\title{
ASYMPTOTIC PROBABILITIES OF AN EXCEEDANCE OVER RENEWAL THRESHOLDS WITH AN APPLICATION TO RISK THEORY
}

\author{
CHRISTIAN Y. ROBERT, ${ }^{*}$ CNAM and CREST
}

\begin{abstract}
Let $\left(Y_{n}, N_{n}\right)_{n \geq 1}$ be independent and identically distributed bivariate random variables such that the $N_{n}$ are positive with finite mean $v$ and the $Y_{n}$ have a common heavy-tailed distribution $F$. We consider the process $\left(Z_{n}\right)_{n \geq 1}$ defined by $Z_{n}=Y_{n}-\Sigma_{n-1}$, where $\Sigma_{n-1}=\sum_{k=1}^{n-1} N_{k}$. It is shown that the probability that the $\operatorname{maximum} M=\max _{n \geq 1} Z_{n}$ exceeds $x$ is approximately $v^{-1} \int_{x}^{\infty} \bar{F}(u) \mathrm{d} u$, as $x \rightarrow \infty$, where $\bar{F}:=1-F$. Then we study the integrated tail of the maximum of a random walk with long-tailed increments and negative drift over the interval $[0, \sigma]$, defined by some stopping time $\sigma$, in the case in which the randomly stopped sum is negative. Finally, an application to risk theory is considered.
\end{abstract}

Keywords: Renewal process; maximum of a random walk; regenerative process; stopping time; heavy-tailed distribution; ruin probability; risk theory

2000 Mathematics Subject Classification: Primary 60G70

Secondary 60G50; 60G40; 60K30

\section{Introduction}

Throughout this paper, $(Y, N)$ and $\left(Y_{n}, N_{n}\right)_{n \geq 1}$ are independent and identically distributed (i.i.d.) bivariate random variables (RVs) such that $N$ is positive with finite mean $v$ and $Y$ has a heavy-tailed distribution $F$. We consider the process $\left(Z_{n}\right)_{n \geq 1}$ defined by $Z_{n}=Y_{n}-\Sigma_{n-1}$, where $\Sigma_{n-1}=\sum_{k=1}^{n-1} N_{k}$ (and $\Sigma_{0}=0$ ), and study the probability that the maximum $M=$ $\max _{n \geq 1} Z_{n}$ exceeds large $x$. This is also the probability that there exists an $n$ such that $Y_{n}$ exceeds $x+\Sigma_{n-1}$, where $\left(\Sigma_{n-1}\right)_{n \geq 1}$ is a sequence of renewal thresholds. The renewal threshold $\Sigma_{n-1}$ is independent of $Y_{n}$, but can be a function of its past values. It is shown that this probability is approximately $v^{-1} \int_{x}^{\infty} \bar{F}(u) \mathrm{d} u$ for large $x$, where $\bar{F}:=1-F$. (Throughout the paper, this notation holds for a general distribution.)

A possible application of this result is to risk theory. Let us consider the following risk model with renewal arrivals:

(i) the claim sizes $U_{1}, U_{2}, \ldots$ are i.i.d. positive RVs with mean $\mu_{U}$;

(ii) the claims happen at random times $s_{1}<s_{2}<\cdots$ such that $T_{n}=s_{n}-s_{n-1}$ are i.i.d. positive RVs with mean $\mu_{T}$, and are independent of $\left(U_{n}\right)_{n \geq 1}$;

(iii) the premium rate is assumed to be equal to 1 and $\mu_{U}<\mu_{T}$.

We define the claim surplus process and the risk reserve process by $S_{t}^{\mathrm{c}}=\sum_{k=1}^{N_{t}} U_{k}-t$ and $R_{t}=-S_{t}^{\mathrm{c}}$, respectively, where $N_{t}=\max \left\{n \geq 0: s_{n} \leq t\right\}$. Let $u$ be the initial solvency

Received 17 March 2004; revision received 1 September 2004.

* Postal address: Centre de Recherche en Economie et Statistique, Timbre J320, 15 Boulevard Gabriel Peri, 92245

Malakoff Cedex, France. Email address: chrobert@ensae.fr 
margin, which is met by capital provided by the shareholders. The classical probability of ultimate ruin $\psi(u)$ is the probability that the claim surplus process ever exceeds the level $u$, i.e. $\mathrm{P}\left(\max _{t \geq 0} S_{t}^{\mathrm{c}}>u\right)$. Since ruin can only occur at claim times, the ruin probability is equal to $\mathrm{P}\left(\max _{n \geq 0} S_{n}>u\right)$, where $\left(S_{n}\right)_{n \geq 1}$ is the random walk with increments $X_{n}=U_{n}-T_{n}$.

A reasonable modification of this model is that some dividends are paid out to the shareholders when the reserve process is sufficiently large. Dividend barrier models have a long history in risk theory (Bühlmann (1996)), but other situations can be considered. Let $\varphi: \mathbb{R}^{+} \rightarrow \mathbb{R}^{+}$be a positive function such that $0 \leq \varphi(x) \leq x$. The insurance company uses a stopping time $\sigma_{1}$ to decide when, just after a claim, the reserves $-S_{\sigma_{1}}$ are sufficiently large (and necessarily positive) that a part $\varphi\left(-S_{\sigma_{1}}\right)$ is distributed to the shareholders and the other part $-S_{\sigma_{1}}-\varphi\left(-S_{\sigma_{1}}\right)$ kept to reinforce the solvency margin. At this time, the reserves are reduced to 0 . The same rule is then used to define a sequence of stopping times $\sigma_{k}$ and a sequence of dividends $\varphi\left(S_{\sigma_{k-1}}-S_{\sigma_{k}}\right)$. Let us define the process $\left\{S_{n}^{\varphi}\right\}$ by

$$
S_{n}^{\varphi}=S_{n}+\sum_{\left\{k \geq 1: \sigma_{k}<n\right\}} \varphi\left(S_{\sigma_{k-1}}-S_{\sigma_{k}}\right),
$$

where $\sigma_{0}=0$ and $S_{0}=0$. The probability of ruin is thus equal to $\psi(u)=\mathrm{P}\left(M^{\varphi}>u\right)$, where $M^{\varphi}=\max \left\{S_{n}^{\varphi}: n \geq 1\right\}$. If we set $Y_{n}=\max _{0 \leq k<\sigma_{n}-\sigma_{n-1}}\left(S_{k+\sigma_{n-1}}-S_{\sigma_{n-1}}\right)$ and $N_{n}=\rho\left(S_{\sigma_{n-1}}-S_{\sigma_{n}}\right)$, where $\rho(x)=x-\varphi(x)$, then it is easy to see that $\mathrm{P}\left(M^{\varphi}>u\right)=$ $\mathrm{P}(M>u)$. According to our result, the probability of ruin is approximately

$$
\psi(u) \sim \mathrm{E}\left(\rho\left(-S_{\sigma_{1}}\right)\right)^{-1} \int_{u}^{\infty} \mathrm{P}(Y>x) \mathrm{d} x \text { for large } u .
$$

We shall not solve the problem of optimal dividend payment under a ruin constraint. (See Bühlmann (1996) and references given there, as well as Gerber (1979), for such a problem.)

In a recent paper, Foss and Zachary (2003) studied the tail behavior of the maximum of a random walk with long-tailed increments $\left(X_{n}\right)$ and negative drift over the interval $[0, \sigma]$ defined by some stopping time $\sigma$. Those authors showed that

$$
\mathrm{P}(Y>x) \sim \mathrm{E} \sigma \mathrm{P}(X>x) \text { for large } x,
$$

where $X$ has the same distribution as the $X_{n}$. In the case in which the randomly stopped sum is negative, we derive the similar equivalence

$$
\int_{u}^{\infty} \mathrm{P}(Y>x) \mathrm{d} x \sim \mathrm{E} \sigma \int_{u}^{\infty} \mathrm{P}(X>x) \mathrm{d} x \text { for large } u,
$$

as a corollary of our result. Then the probability of ruin is approximately

$$
\psi(u) \sim \mathrm{E}\left(\rho\left(-S_{\sigma_{1}}\right)\right)^{-1} \mathrm{E} \sigma_{1} \int_{u}^{\infty} \mathrm{P}(X>x) \mathrm{d} x \text { for large } u .
$$

In the case in which no dividend is distributed, $\varphi$ is equal to 0 and, by using Wald's identity (Chow et al. (1965)), $\mathrm{E}\left(\rho\left(-S_{\sigma_{1}}\right)\right)=-\mathrm{E} S_{\sigma_{1}}=-\mathrm{E} \sigma_{1} \mathrm{E} X$, which gives the classical asymptotics for the probability of ruin.

In Section 2, we present the results of the paper. All the proofs are postponed to Section 3. 


\section{Results}

We first state our main result, which is Theorem 2.1. We then give Corollary 2.1, Corollary 2.2, and Proposition 2.1, which set out the conditions for the application to risk theory.

Theorem 2.1. Let us assume that $\mathrm{E} N^{2}<\infty$ and that

$$
\lim _{x \rightarrow \infty} \frac{\mathrm{P}(Y>x)}{\int_{x}^{\infty} \mathrm{P}(Y>u) \mathrm{d} u}=0 .
$$

Then,

$$
\lim _{x \rightarrow \infty} \frac{\mathrm{P}(M>x)}{\int_{x}^{\infty} \mathrm{P}(Y>u) \mathrm{d} u}=\frac{1}{\mathrm{E} N} .
$$

Remark 2.1. Equation (2.1) is not a condition ordinarily used to define heavy-tailed distributions. A more usual condition is that the distribution of $Y$ be long tailed, i.e.

$$
\lim _{x \rightarrow \infty} \frac{\mathrm{P}(Y>x-h)}{\mathrm{P}(Y>x)}=1 \text { for all fixed } h>0
$$

(see below for other definitions). If $Y$ is long tailed then it is easy to see that condition (2.1) holds.

Remark 2.2. Let $\left(S_{n}\right)_{n \geq 1}$ be a random walk with heavy-tailed increments $X_{n}$ and negative mean. It is well known that

$$
\lim _{x \rightarrow \infty} \frac{\mathrm{P}\left(\max _{n \geq 1} S_{n}>x\right)}{\int_{x}^{\infty} \mathrm{P}(X>u) \mathrm{d} u}=-\frac{1}{\mathrm{E} X}
$$

(see, e.g. Veraverbeke (1977), Embrechts and Veraverbeke (1982), and Korshunov (1997)). Theorem 2.1 seems to hold with $Y_{n}=X_{n}$ and $N_{n}=-X_{n}$, although the $-X_{n}$ are not almost surely positive, and have a positive mean.

Now consider a regenerative process $\left\{V_{n}\right\}_{n \geq 0}$ : there exists a zero-delayed renewal process with epochs $T_{0}=0<T_{1}<T_{2}<\cdots$ such that the cycles $\left\{V_{n+T_{k-1}}\right\}_{0 \leq n<T_{k}-T_{k-1}}$ are independent and have the same distribution. Regenerative processes have many important applications in queueing networks, storage processes, insurance, and finance (see, e.g. Asmussen (1987), (2000)). A basic example is the Lindley process and its cycles, that is, the time intervals separated by the instants at which the process is equal to 0 . We write $c_{k}=T_{k}-T_{k-1}$ for the cycle lengths and let $c$ be an RV with the same distribution as the $c_{k}$. We define $\kappa=\mathrm{E} c$, and assume this to be finite. Let us define the maxima over cycles as

$$
M_{c_{k}}=\max \left\{V_{n+T_{k-1}}: 0 \leq n<T_{k}-T_{k-1}\right\} .
$$

Furthermore, let us assume that there exist RVs $N_{n}$ such that $\left(\left\{V_{n+T_{k-1}}\right\}_{0 \leq n<T_{k}-T_{k-1}}, N_{k}\right)_{k \geq 1}$ are i.i.d. As a consequence of Theorem 2.1, we give the asymptotic tail behavior of $M=$ $\max _{k \geq 1}\left(M_{c_{k}}-\Sigma_{k-1}\right)$, i.e. the probability that the regenerative process exceeds increasingly high thresholds that are defined by a renewal process and are constant on regenerative cycles.

Corollary 2.1. Let us assume that $\mathrm{E} N^{2}<\infty$ and that

$$
\lim _{x \rightarrow \infty} \frac{\mathrm{P}\left(M_{c}>x\right)}{\int_{x}^{\infty} \mathrm{P}\left(M_{c}>u\right) \mathrm{d} u}=0 .
$$


Then,

$$
\lim _{x \rightarrow \infty} \frac{\mathrm{P}(M>x)}{\int_{x}^{\infty} \mathrm{P}\left(M_{c}>u\right) \mathrm{d} u}=\frac{1}{\mathrm{E} N} .
$$

The proof is omitted because it is a direct application of Theorem 2.1.

Let $\left\{X_{n}\right\}_{n \geq 1}$ be a sequence of i.i.d. random variables. By $F_{X}$ we denote the common distribution of the $X_{n}$, and we assume that $\mu=\mathrm{E} X_{n}<0$ and $\mathrm{P}\left(X_{1}>0\right)>0$. We consider a stopping time $\sigma$ with respect to the filtration $\left\{\mathcal{F}_{n}\right\}_{n \geq 1}$, where $\mathcal{F}_{n}=\sigma\left(X_{1}, \ldots, X_{n}\right)$, and write $S_{n}=\sum_{i=1}^{n} X_{i}$ and $M_{\sigma}=\max \left\{S_{n}: n=0,1, \ldots, \sigma-1\right\}$.

Before stating Corollary 2.2, we first provide some further definitions. For any distribution function $H$ on $\mathbb{R}$ with finite mean, we define the integrated distribution function $H^{s}$ by $\overline{H^{s}}=\min \left(1, \int_{x}^{\infty} \bar{H}(u) \mathrm{d} u\right)$. A distribution function $H$ on $\mathbb{R}^{+}$is subexponential if and only if $H(x)>0$ for all $x$ and $\lim _{x \rightarrow \infty} \overline{H^{* n}}(x) / \bar{H}(x)=n, n \geq 2$, where $H^{* n}$ is the $n$-fold convolution of $H$ with itself. (It is sufficient that only the first of these conditions holds in the case $n=2$.) More generally, a distribution function $H$ on $\mathbb{R}$ is subexponential if and only if $H^{+}$ is subexponential, where $H^{+}=H \mathbf{1}_{\mathbb{R}^{+}}$and $\mathbf{1}_{\mathbb{R}^{+}}$is the indicator function of $\mathbb{R}^{+}$. A well-known result is that subexponential distributions are long tailed. Finally, a distribution function $H$ on $\mathbb{R}$ belongs to the class $8^{*}$ if and only if $H(x)>0$ for all $x$ and

$$
\lim _{x \rightarrow \infty} \frac{\int_{0}^{x} \bar{H}(x-y) \bar{H}(y) \mathrm{d} y}{\bar{H}(x)}=\int_{0}^{\infty} \bar{H}(y) \mathrm{d} y .
$$

It is also known that if $H \in 8^{*}$, then $H$ and $H^{s}$ are subexponential (see Klüppelberg (1988)).

Corollary 2.2. (i) Suppose that $F_{X} \in 8^{*}$. Let $\sigma$ be any stopping time such that $\mathrm{P}\left(S_{\sigma} \leq 0\right)=1$ and $\mathrm{E} S_{\sigma}^{2}<\infty$. Then

$$
\lim _{x \rightarrow \infty} \frac{\int_{x}^{\infty} \mathrm{P}\left(M_{\sigma}>u\right) \mathrm{d} u}{\int_{x}^{\infty} \bar{F}_{X}(u) \mathrm{d} u}=\mathrm{E} \sigma .
$$

(ii) Suppose that (2.2) holds for some stopping time $\sigma$ such that $\mathrm{P}\left(S_{\sigma} \leq 0\right)=1$ and $\mathrm{E} S_{\sigma}^{2}<\infty$. Then, $F_{X}^{S}$ is subexponential.

Remark 2.3. If we assume that $\mathrm{E}\left(X_{1}^{+}\right)^{2}<\infty$ and $\mathrm{E} \sigma^{2}<\infty$, then $\mathrm{E} S_{\sigma}^{2}<\infty$ (see Gut and Janson (1986), Theorem 3.1). Moreover, if the stopping time is the first-passage time $\min \left\{n: S_{n}<c\right\}$, with $c$ a nonpositive constant, then the condition $\mathrm{E}\left(X_{1}^{+}\right)^{2}<\infty$ implies that $\mathrm{E} \sigma^{2}<\infty$, from Theorem 2.1 of Gut (1974). In this case, only the condition $\mathrm{E}\left(X_{1}^{+}\right)^{2}<\infty$ is needed.

Finally, we come back to the risk problem outlined in the Introduction. We found that, as in the classical risk model, the probability of ruin is equivalent to the integrated tail of $X$.

Proposition 2.1. Suppose that $F_{X} \in 8^{*}$. Then,

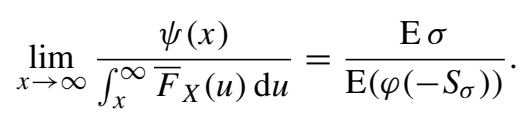




\section{Proofs}

We first state a lemma that is needed for the proof of Theorem 2.1.

Lemma 3.1. Let $\left\{\xi_{n}\right\}_{n \geq 1}$ be a sequence of i.i.d. $R V$ s with $\mathrm{E} \xi_{1}=-m<0$ and $\mathrm{P}\left(\xi_{1}>0\right)>0$. Let us define $\xi_{1}^{+}=\max \left(0, \xi_{1}\right)$. Then,

$$
\mathrm{E}\left(\xi_{1}^{+}\right)^{2}<\infty \Leftrightarrow \sum_{n \geq 1} \mathrm{P}\left(\sum_{i=1}^{n} \xi_{i}>0\right)<\infty .
$$

Proof. Consider the probability that $n$ is the epoch of the first entry into $]-\infty, 0[$ of the random walk with increments $\left\{\xi_{n}\right\}$, that is,

$$
\tau_{n}^{\xi}=\mathrm{P}\left(\xi_{1} \geq 0, \ldots, \sum_{i=1}^{n-1} \xi_{i} \geq 0, \sum_{i=1}^{n} \xi_{i}<0\right) .
$$

Then, $\left\{\tau_{n}^{\xi}\right\}$ is the distribution of the first descending ladder epoch $\tau_{-}^{\xi}$. The finiteness of the moments of $\tau_{-}^{\xi}$ is equivalent to the finiteness of the moments of $\xi_{1}^{+}$(see Gut (1974), Theorem 2.1): if we let $r \geq 1$ then $\mathrm{E}\left(\left|\xi_{1}^{+}\right|\right)^{r}<\infty$ if and only if $\mathrm{E}\left(\tau_{-}^{\xi}\right)^{r}<\infty$.

The probability-generating function of $\left\{\tau_{n}^{\xi}\right\}$ is given by $P^{\xi}(s)=\sum_{n \geq 1} \tau_{n}^{\xi} s^{n}, 0 \leq s \leq 1$. The Sparre-Anderson theorem (see Feller (1971, Theorem XII.7.1)) establishes that

$$
\log \frac{1-P^{\xi}(s)}{1-s}=\sum_{n \geq 1} \frac{s^{n}}{n} \mathrm{P}\left(\sum_{i=1}^{n} \xi_{i}>0\right), \quad 0 \leq s<1 .
$$

Since, by assumption, $\mathrm{E}\left|\xi_{1}^{+}\right|<\infty$, we have $\mathrm{E} \tau_{-}^{\xi}<\infty$ and we can introduce the following probability-generating function:

$$
Q^{\xi}(s)=\frac{1}{\mathrm{E} \tau_{-}^{\xi}} \frac{1-P^{\xi}(s)}{(1-s)}, \quad 0 \leq s \leq 1 .
$$

This is the probability-generating function of the random variable $I_{\tau_{-}^{\xi}}$ defined, for $j \geq 0$, by $\mathrm{P}\left(I_{\tau_{-}^{\xi}}=j\right)=\sum_{k>j} \tau_{j}^{\xi} / \mathrm{E} \tau_{-}^{\xi}$ (see Feller (1970, p. 265)). By differentiating (3.1), we have

$$
\frac{\left(Q^{\xi}\right)^{\prime}(s)}{Q^{\xi}(s)}=\sum_{n \geq 1} s^{n-1} \mathrm{P}\left(\sum_{i=1}^{n} \xi_{i}>0\right), \quad 0 \leq s<1,
$$

from which it follows that

$$
\begin{aligned}
\lim _{s \nearrow 1}\left(Q^{\xi}\right)^{\prime}(s)<\infty & \Leftrightarrow \sum_{n \geq 1} \mathrm{P}\left(\sum_{i=1}^{n} \xi_{i}>0\right)<\infty \\
& \Leftrightarrow \mathrm{E} I_{\tau_{-}^{\xi}}<\infty \Leftrightarrow \mathrm{E}\left(\tau_{-}^{\xi}\right)^{2}<\infty .
\end{aligned}
$$

We conclude that $\mathrm{E}\left(\left|\xi_{1}^{+}\right|\right)^{2}<\infty$ is equivalent to $\sum_{n \geq 1} \mathrm{P}\left(\sum_{i=1}^{n} \xi_{i}>0\right)<\infty$. 


\subsection{Proof of Theorem 2.1}

Let us write $M^{k}=\max _{1 \leq i \leq k}\left(Y_{k}-\Sigma_{k-1}\right)$. Then

$$
\mathrm{P}\left(M^{k}>x\right)=\mathrm{P}\left(\bigcup_{i=1}^{k}\left\{Y_{i}>x+\Sigma_{i-1}\right\}\right)
$$

The proof of Theorem 2.1 is based on several steps.

Step 1: an upper bound for $\mathrm{P}(M>x)$. Since $\mathrm{P}\left(M^{k}>x\right)$ is bounded by $\sum_{i=1}^{k} \mathrm{P}\left(Y_{i}>\right.$ $\left.x+\Sigma_{i-1}\right)$, we focus our attention on the probability

$$
\begin{aligned}
\mathrm{P}\left(Y_{i}>x+\Sigma_{i-1}\right)= & \mathrm{P}\left(Y_{i}>x+\Sigma_{i-1}, \Sigma_{i-1}<(i-1) v(1-\varepsilon)\right) \\
& +\mathrm{P}\left(Y_{i}>x+\Sigma_{i-1}, \Sigma_{i-1} \geq(i-1) v(1-\varepsilon)\right) \\
\leq & \mathrm{P}\left(Y_{i}>x, \Sigma_{i-1}<(i-1) v(1-\varepsilon)\right)+\mathrm{P}\left(Y_{i} \geq x+(i-1) v(1-\varepsilon)\right) \\
\leq & \mathrm{P}\left(Y_{i}>x\right) \mathrm{P}\left(\Sigma_{i-1}<(i-1) v(1-\varepsilon)\right)+\mathrm{P}\left(Y_{i} \geq x+(i-1) v(1-\varepsilon)\right) .
\end{aligned}
$$

It follows that

$$
\mathrm{P}\left(M^{k}>x\right) \leq \bar{F}(x) \sum_{i=1}^{k} \mathrm{P}\left(\tilde{\Sigma}_{i-1}^{u}>0\right)+\sum_{i=1}^{k} \bar{F}(x+(i-1) v(1-\varepsilon)),
$$

where $\tilde{\Sigma}_{i-1}^{u}=(i-1) v(1-\varepsilon)-\Sigma_{i-1}$. From Lemma 3.1 and the facts that $\mathrm{E} \tilde{\Sigma}_{1}^{u}<0$ and $\mathrm{E}(N)^{2}<\infty$, we deduce that

$$
\sum_{i=1}^{k} \mathrm{P}\left(\tilde{\Sigma}_{i-1}^{u}>0\right)<\sum_{i=1}^{\infty} \mathrm{P}\left(\tilde{\Sigma}_{i-1}^{u}>0\right)=: K_{1}(\varepsilon)<\infty
$$

and

$$
\mathrm{P}\left(M^{k}>x\right) \leq \bar{F}(x) K_{1}(\varepsilon)+\sum_{i=1}^{k} \bar{F}(x+(i-1) v(1-\varepsilon)) .
$$

Then, as $k \rightarrow \infty$, we have $\mathrm{P}\left(M^{k}>x\right) \nearrow \mathrm{P}(M>x)$ and, so,

$$
\begin{aligned}
\mathrm{P}(M>x) & \leq \bar{F}(x) K_{1}(\varepsilon)+\sum_{i=1}^{\infty} \bar{F}(x+(i-1) v(1-\varepsilon)) \\
& \leq \frac{\int_{x}^{\infty} \bar{F}(u) \mathrm{d} u}{v(1-\varepsilon)}+\bar{F}(x)\left(K_{1}(\varepsilon)+1\right)
\end{aligned}
$$

Step 2: a lower bound for $\mathrm{P}(M>x)$. First, let us note that

$$
\mathrm{P}(M>x) \geq \sum_{i=1}^{\infty} \mathrm{P}\left(Y_{i}>x+\Sigma_{i-1}\right)-\sum_{1 \leq i<j} \mathrm{P}\left(Y_{i}>x+\Sigma_{i-1}, Y_{j}>x+\Sigma_{j-1}\right) .
$$


On one hand, we have

$$
\begin{aligned}
\mathrm{P}\left(Y_{i}>x+\Sigma_{i-1}\right) \geq & \mathrm{P}\left(Y_{i}>x+\Sigma_{i-1}, \Sigma_{i-1} \leq(i-1) v(1+\varepsilon)\right) \\
\geq & \mathrm{P}\left(Y_{i}>x+(i-1) v(1+\varepsilon), \Sigma_{i-1} \leq(i-1) v(1+\varepsilon)\right) \\
= & \mathrm{P}\left(Y_{i}>x+(i-1) v(1+\varepsilon)\right) \mathrm{P}\left(\Sigma_{i-1} \leq(i-1) v(1+\varepsilon)\right) \\
= & \mathrm{P}\left(Y_{i}>x+(i-1) v(1+\varepsilon)\right) \\
& -\mathrm{P}\left(Y_{i}>x+(i-1) v(1+\varepsilon)\right) \mathrm{P}\left(\Sigma_{i-1}>(i-1) v(1+\varepsilon)\right) \\
\geq & \mathrm{P}\left(Y_{i}>x+(i-1) v(1+\varepsilon)\right)-\mathrm{P}\left(Y_{i}>x\right) \mathrm{P}\left(\Sigma_{i-1}>(i-1) v(1+\varepsilon)\right) .
\end{aligned}
$$

It follows, from Lemma 3.1 and $\mathrm{E}(N)^{2}<\infty$, that $K_{2}(\varepsilon):=\sum_{i=1}^{\infty} \mathrm{P}\left(\tilde{\Sigma}_{i-1}^{l}>0\right)<\infty$, where $\tilde{\Sigma}_{i-1}^{l}=\Sigma_{i-1}-(i-1) v(1+\varepsilon)$ since $\mathrm{E} \tilde{\Sigma}_{1}^{l}<0$. Therefore,

$$
\begin{aligned}
\sum_{i=1}^{\infty} \mathrm{P}\left(Y_{i}>x+\Sigma_{i-1}\right) & \geq \sum_{i=1}^{\infty} \bar{F}(x+(i-1) v(1+\varepsilon))-\bar{F}(x) K_{2}(\varepsilon) \\
& \geq \frac{\int_{x}^{\infty} \bar{F}(u) \mathrm{d} u}{v(1+\varepsilon)}-\bar{F}(x) K_{2}(\varepsilon) .
\end{aligned}
$$

On the other hand, let us write $\Sigma_{i+1}^{j-1}=\sum_{k=i+1}^{j-1} N_{k}$ for $i<j$. We have

$$
\begin{aligned}
\mathrm{P}\left(Y_{i}>x+\Sigma_{i-1}, Y_{j}>x+\Sigma_{j-1}\right) & \leq \mathrm{P}\left(Y_{i}>x+\Sigma_{i-1}, Y_{j}>x+\Sigma_{i+1}^{j-1}\right) \\
& \leq \mathrm{P}\left(Y_{i}>x+\Sigma_{i-1}\right) \mathrm{P}\left(Y_{j}>x+\Sigma_{i+1}^{j-1}\right) \\
& =\mathrm{P}\left(Y_{i}>x+\Sigma_{i-1}\right) \mathrm{P}\left(Y_{j}>x+\Sigma_{j-i-1}\right) .
\end{aligned}
$$

In analogy to step 1 ,

$$
\sum_{1 \leq i<j} \mathrm{P}\left(Y_{i}>x+\Sigma_{i-1}, Y_{j}>x+\Sigma_{j-1}\right) \leq\left(\frac{\int_{x}^{\infty} \bar{F}(u) \mathrm{d} u}{v(1-\varepsilon)}+\bar{F}(x)\left(K_{1}(\varepsilon)+1\right)\right)^{2} .
$$

Thus, a lower bound is given by

$$
\mathrm{P}(M>x) \geq \frac{\int_{x}^{\infty} \bar{F}(u) \mathrm{d} u}{\nu(1+\varepsilon)}-\bar{F}(x) K_{2}(\varepsilon)-\left(\frac{\int_{x}^{\infty} \bar{F}(u) \mathrm{d} u}{v(1-\varepsilon)}+\bar{F}(x)\left(K_{1}(\varepsilon)+1\right)\right)^{2} .
$$

Step 3. Let us use steps 1 and 2 and let $x \rightarrow \infty$. Condition (2.1) then implies that

$$
\frac{(1-\varepsilon)}{\mathrm{E} N} \leq \lim _{x \rightarrow \infty} \frac{\mathrm{P}(M>x)}{\int_{x}^{\infty} \mathrm{P}(Y>u) \mathrm{d} u} \leq \frac{(1+\varepsilon)}{\mathrm{E} N} .
$$

To complete the proof, we let $\varepsilon \rightarrow 0$.

\subsection{Proof of Corollary 2.2}

Step 1. Let us define the sequence of stopping times $\left\{\sigma_{k}\right\}_{k \geq 0}$ such that $\sigma_{0}=0$ and $\sigma_{1}=\sigma$, and the cycles

$$
\left\{S_{n+\sigma_{k-1}}-S_{\sigma_{k-1}}\right\}_{0 \leq n<\sigma_{k}-\sigma_{k-1}},
$$


which are independent and have the same distribution for $k=1,2, \ldots$ Let us write $V_{n+\sigma_{k-1}}=$ $S_{n+\sigma_{k-1}}-S_{\sigma_{k-1}}$ for $0 \leq n<\sigma_{k}-\sigma_{k-1}$, and $N_{k}=S_{\sigma_{k-1}}-S_{\sigma_{k}}$. By the regenerative structure of the random walk, $\left(\left\{V_{n+\sigma_{k-1}}\right\}_{0 \leq n \leq \sigma_{k}-\sigma_{k-1}}, N_{k}\right)_{k \geq 1}$ are i.i.d. We remark that

$$
M=\max _{n \geq 1} S_{n}=\max _{k \geq 1} \max _{0 \leq n<\sigma_{k}-\sigma_{k-1}}\left(V_{n+\sigma_{k-1}}-\Sigma_{k-1}\right) .
$$

Since $F_{X}^{s}$ is subexponential, Veraverbeke's theorem (Korshunov (1997)) implies that

$$
\lim _{x \rightarrow \infty} \frac{\mathrm{P}(M>x)}{\int_{x}^{\infty} \bar{F}_{X}(u) \mathrm{d} u}=-\frac{1}{\mu}
$$

and, since $\mathrm{E}(N)^{2}=\mathrm{E}\left(-S_{\sigma}\right)^{2}<\infty$, in order to apply Corollary 2.1, it suffices to verify that

$$
\lim _{x \rightarrow \infty} \frac{\mathrm{P}\left(M_{\sigma}>x\right)}{\int_{x}^{\infty} \mathrm{P}\left(M_{\sigma}>u\right) \mathrm{d} u}=0 .
$$

Let us define the sequence of stopping times $\left\{\tau_{k}\right\}_{k \geq 0}$ by

$$
\tau_{0}=0, \quad \tau_{k}=\min \left\{n: n>\tau_{k-1}, S_{n} \leq S_{\tau_{k-1}}\right\},
$$

so that $\tau_{k}$ is the $k$ th descending ladder time. Since $S_{\sigma} \leq 0$ almost surely, it follows that $\tau_{1} \leq \sigma$ almost surely. If we let $\mu(x)=\min \left\{n: S_{n}>x\right\}$, then

$$
\begin{aligned}
\mathrm{P}\left(M_{\sigma}>x\right) & =\sum_{k \geq 1} \mathrm{P}\left(\tau_{k}<\mu(x) \leq \tau_{k+1}, \sigma>\mu(x)\right) \\
& =\sum_{k \geq 1} \mathrm{P}\left(\sigma>\mu(x)>\tau_{k}\right) \mathrm{P}\left(\tau_{k}<\mu(x) \leq \tau_{k+1} \mid \sigma>\mu(x)\right) \\
& \leq \sum_{k \geq 1} \mathrm{P}\left(\sigma>\tau_{k}\right) \mathrm{P}\left(M_{\tau_{k}}>x\right) \leq \sum_{k \geq 1} \mathrm{P}(\sigma>k) \mathrm{P}\left(M_{\tau}>x\right) \\
& \leq \mathrm{E} \sigma \mathrm{P}\left(M_{\tau}>x\right)
\end{aligned}
$$

Moreover,

$$
\mathrm{P}\left(M_{\tau}>x\right) \leq \mathrm{P}\left(\sharp\left\{n<\tau: S_{n}>x, S_{n+1} \leq x\right\} \geq 1\right) \leq \mathrm{E} \sum_{n=0}^{\tau-1} \mathbf{1}_{\left\{S_{n}>x, S_{n+1} \leq x\right\}},
$$

where $\sharp A$ denotes the cardinality of $A$. By using the argument of the proof of Lemma 2.3 of Asmussen (1998), we find that

$$
\mathrm{E} \sum_{n=0}^{\tau-1} \mathbf{1}_{\left\{S_{n}>x, S_{n+1} \leq x\right\}} \sim \mathrm{E} \tau \frac{\mu^{-}}{\mu} \mathrm{P}(X>x) \text { for large } x,
$$

where $\mu^{-}=\int_{0}^{\infty} F_{X}(-y) \mathrm{d} y$. Since $\mathrm{P}\left(M_{\sigma}>x\right) \geq \mathrm{P}(X>x)$, we find that

$$
\frac{\mathrm{P}\left(M_{\sigma}>x\right)}{\int_{x}^{\infty} \mathrm{P}\left(M_{\sigma}>u\right) \mathrm{d} u} \leq \mathrm{E} \sigma \mathrm{E} \tau \frac{\mu^{-}}{\mu} \frac{\mathrm{P}(X>x)}{\int_{x}^{\infty} \mathrm{P}(X>u) \mathrm{d} u} \rightarrow 0
$$

as $x \rightarrow \infty$, since the distribution of $X$ is long tailed. 
By Corollary 2.1, we have

$$
\lim _{x \rightarrow \infty} \frac{\mathrm{P}(M>x)}{\int_{x}^{\infty} \mathrm{P}\left(M_{\sigma}>u\right) \mathrm{d} u}=-\frac{1}{\mathrm{E} S_{\sigma}} .
$$

Moreover, Wald's identity (see Chow et al. (1965)) gives that if either $\mathrm{E}\left|X_{1}\right|<\infty$ or $\mathrm{E} \sigma<\infty$, then $\mathrm{E} S_{\sigma}=\mu \mathrm{E} \sigma$. Now, using Veraverbeke's theorem, we derive that

$$
\lim _{x \rightarrow \infty} \frac{\int_{x}^{\infty} \mathrm{P}\left(M_{\sigma}>u\right) \mathrm{d} u}{\int_{x}^{\infty} \bar{F}(u) \mathrm{d} u}=\mathrm{E} \sigma .
$$

Step 2. That $F^{s}$ is subexponential follows from the converse to Veraverbeke's theorem, proved in Korshunov (1997).

\subsection{Proof of Proposition 2.1}

Let us consider the sequences of stopping times $\left\{\sigma_{k}\right\}_{k \geq 0}$ and cycles

$$
\left\{S_{n+\sigma_{k-1}}-S_{\sigma_{k-1}}\right\}_{0 \leq n<\sigma_{k}-\sigma_{k-1}}
$$

defined in step 1 of Section 3.2. We now write $V_{n+\sigma_{k-1}}=S_{n+\sigma_{k-1}}-S_{\sigma_{k-1}}$ for $0 \leq n<\sigma_{k}-\sigma_{k-1}$ and $k \geq 1, Y_{n}=\max _{0 \leq k<\sigma_{n}-\sigma_{n-1}}\left(S_{k+\sigma_{n-1}}-S_{\sigma_{n-1}}\right)$, and $N_{n}=\rho\left(S_{\sigma_{n-1}}-S_{\sigma_{n}}\right)$. By the regenerative structure of the random walk, $\left(\left\{V_{n+\sigma_{k-1}}\right\}_{0 \leq n \leq \sigma_{k}-\sigma_{k-1}}, N_{k}\right)_{k \geq 1}$ are again i.i.d. and

$$
M^{\varphi}=\max _{n \geq 1} S_{n}^{\varphi}=\max _{k \geq 1} \max _{0 \leq n<\sigma_{k}-\sigma_{k-1}}\left(V_{n+\sigma_{k-1}}-\Sigma_{k-1}\right) .
$$

According to Corollary 2.1,

$$
\lim _{x \rightarrow \infty} \frac{\mathrm{P}\left(M^{\varphi}>x\right)}{\int_{x}^{\infty} \mathrm{P}(Y>u) \mathrm{d} u}=\frac{1}{\mathrm{E} \rho\left(-S_{\sigma_{1}}\right)}
$$

and, according to Corollary 2.2,

$$
\lim _{x \rightarrow \infty} \frac{\int_{x}^{\infty} \mathrm{P}(Y>u) \mathrm{d} u}{\int_{x}^{\infty} \bar{F}_{X}(u) \mathrm{d} u}=\mathrm{E} \sigma_{1} .
$$

These observations complete the proof of Proposition 2.1.

\section{Acknowledgement}

The author wishes to thank an anonymous referee for a careful reading of the paper and detailed comments.

\section{References}

Asmussen, S. (1987). Applied Probability and Queues. John Wiley, Chichester.

Asmussen, S. (1998). Subexponential asymptotics for stochastic processes: extremal behavior, stationary distributions and first passage probabilities. Ann. Appl. Prob. 8, 354-374.

Asmussen, S. (2000). Ruin Probabilities (Adv. Ser. Statist. Sci. Appl. Prob. 2). World Scientific, River Edge, NJ.

Bühlmann, H. (1996). Mathematical Methods of Risk Theory. Springer, Berlin.

Chow, Y. S., Robbins, H. And Teicher, H. (1965). Moments of randomly stopped sums. Ann. Math. Statist. 36, 789-799. 
Embrechts, P. and Veraverbeke, N. (1982). Estimates for the probability of ruin with special emphasis on the possibility of large claims. Insurance Math. Econom. 1, 55-72.

Feller, W. (1970). An Introduction to Probability Theory and Its Applications, Vol. 1, 3rd edn. John Wiley, New York. Feller, W. (1971). An Introduction to Probability Theory and Its Applications, Vol. 2, 2nd edn. John Wiley, New York.

Foss, S. And Zachary, S. (2003). The maximum on a random time interval of a random walk with long-tailed increments and negative drift. Ann. Appl. Prob. 13, 37-53.

Gerber H. U. (1979). An Introduction to Mathematical Risk Theory (S. S. Huebner Foundation Monogr. Ser. 8). University of Pennsylvania, Philadelphia, PA.

Gut, A. (1974). On the moments and limit distributions of some first passages times. Ann. Prob. 2, 277-306.

Gut, A. AND JANSON, S. (1986). Converse results for existence of moments and uniform integrability for stopped random walks. Ann. Prob. 14, 1296-1317.

KlÜPPelberG, C. (1988). Subexponential distributions and integrated tails. J. Appl. Prob. 35, 325-347.

Korshunov, D. A. (1997). On distribution tail of the maximum of a random walk. Stoch. Process. Appl. 72, 97-103.

Veraverbeke, N. (1977). Asymptotic behavior of Wiener-Hopf factors of a random walk. Stoch. Process. Appl. 5, 27-37. 